\title{
Factores socioeconómicos asociados a la diversidad pecuaria del traspatio en comunidades mayas de Yucatán y Campeche
}

\author{
Socioeconomic factors associated with backyard diversity livestock in mayan communities of Yucatan \\ and Campeche
}

\author{
Astrid Nohely Aguilar Jiménez' ${ }^{1}$, Miguel Ángel Magaña Magaña1*, Lucely Carminia Contreras UC² \\ División de Estudios de Posgrado e Investigación. Instituto Tecnológico de Conkal. Avenida Tecnológico s/n, domicilio \\ conocido. C.P. 97345. Conkal, Yucatán, México. \\ 2 Estudiante de Doctorado en El Colegio de Sonora. Avenida Obregón No. 54, Col. Centro. C.P. 83000. Hermosillo, Sonora, \\ México.
}

\section{RESUMEN}

El objetivo del presente trabajo fue analizar la influencia de los principales factores socioeconómicos de la familia sobre la diversidad y estructura del inventario de especies pecuarias criadas en el traspatio. Se entrevistó un total de 210 jefas de familias en cuatro comunidades mayas, dos ubicadas en el estado de Yucatán y dos en Campeche, la encuesta por muestreo estadístico se realizó de marzo a junio del 2017 y en ésta se empleó una cédula de entrevista; se estimaron parámetros de dominancia y riqueza específica de especies, la relación entre variables se midió a través de la correlación de Pearson. Los resultados evidencian que la diversidad animal en el agroecosistema familiar depende principalmente del tamaño del predio, edad y escolaridad de la jefa de familia y de la migración; se encontró la dominancia de una especie representada por las aves de corral, mismas que aprovechan mejor los recursos disponibles, su producción es continua y forma parte de la tradición gastronómica y cultural. Se concluye que la cría de animales en las comunidades mayas, principalmente aves de corral y cerdos, es una actividad a baja escala y de subsistencia que contribuye a satisfacer parte de las necesidades básicas de alimentación y genera ingresos y ahorros adicionales.

Palabras clave. Producción pecuaria, Agroecosistema tradicional, Recursos zoogenéticos, Medio rural.

\section{ABSTRACT}

The objective of the present work was to analyze the influence of the families main socioeconomic factors on the diversity and structure of the inventory of livestock species reared in the backyard. A total of 210 woman heads of family were interviewed in four Mayan communities, two located in the state of Yucatan and two in Campeche. The survey by statistical sampling was conducted from March to June 2017 using an interview card. Dominance parameters and species specific richness were estimated, the relationship between variables was measured through the Pearson correlation. The results show that the animal diversity in the family agroecosystem depends mainly on the size of the backyard, age and schooling of women head of family and migration; it was found a dominance of poultry, which take better advantage

*Autor para correspondencia: Miguel Ángel Magaña Magaña

Correo electrónico: drmmagana@hotmail.com of available resources, its production is continuous and is part of the gastronomic and cultural tradition. It is concluded that the breeding of livestock animals in the mayan communities, mainly poultry and pigs, is a low-scale activity and subsistence that contributes to satisfy part of the basic needs of food and generates additional income and savings.

Keywords. Livestock production, Traditional agroecosystem, Animal genetic resources, Rural environment.

\section{INTRODUCCIÓN}

En términos generales, el traspatio o solar se considera como un agroecosistema tradicional, ya que en éste las familias del medio rural han cultivado por generaciones diversas especies vegetales y criado determinadas especies animales (Mariaca, 2012; Gonzáles et al., 2014). Esta área se encuentra ubicada en los alrededores de la casa-habitación donde reside la unidad doméstica campesina y en ella se llevan a cabo diversas actividades entre las que figuran las productivas, recreativas y de convivencia social, en la cual cada uno de los integrantes de la familia participa en su desarrollo (Aké et al., 2002; Cuanalo y Guerra, 2008; Guarneros et al., 2014).La actividad agrícola y pecuaria del traspatio, acorde a su espacio y a la disponibilidad de recursos naturales, se caracteriza por su limitada capacidad productiva o de pequeña escala. En la actividad agrícola se cultivan solo una o algunas plantas por especie, mientras que en la cría de animales se maneja un mayor número de individuos por especie; por ejemplo, en el traspatio de las familias se pueden encontrar diversos animales como gallinas, guajolotes, patos, conejos, cerdos, ovinos y bovinos (Gutiérrez et al., 2012). Así, de la actividad pecuaria se obtienen productos como carne, huevo, leche, entre otros, mismos que son utilizados para el autoconsumo y en parte para el fortalecimiento de la economía familiar, ya que a través de la venta de los excedentes de producción se puede disponer de recursos económicos (Olvera et al., 2017).

La extensa gama de recursos naturales disponibles en el traspatio, las prácticas agroecológicas y la adaptación al medio de las especies animales, fortalecen la sostenibilidad y autosuficiencia de la producción de alimentos. Desde el punto de vista ecológico, lo anterior es importante debido a que da como resultado que las familias conserven una 
amplia diversidad de especies de animales domésticos en sus unidades de producción (Gliessman, 1990; Vargas et al., 2017). Pero factores sociales como la edad, posición o estatus económico, nivel de escolaridad, entre otros, condicionan de manera significativa la forma en la que se maneja la diversidad de especies pecuarias criadas en el agroecosistema tradicional o traspatio.

Por otra parte, el contexto socioeconómico que se vive en las comunidades rurales, obliga que día a día los habitantes de éstas tengan la necesidad de incorporarse al trabajo asalariado en los centros urbanos. Esta migración ocasiona no solo una disminución de la mano de obra para la realización de las actividades productivas, sino que también conduce a un debilitamiento del conocimiento empírico, debido a la pérdida de continuidad en la transmisión de la cultura productiva de una generación a otra y una reducción en la estructura del inventario de las especies domésticas criadas en los traspatios (Guerra, 2005; Salazar et al., 2015).

En resumen, las características socioeconómicas de las familias, la migración hacia los centros urbanos, la necesidad de proteína de origen animal en la alimentación humana y la perdida de diversidad de los recursos zoogenéticos, son motivo de estudio integral. Por lo anterior el objetivo del presente trabajo fue analizar el grado de asociación que se establece entre los principales factores socioeconómicos de la unidad doméstica sobre la diversidad y estructura del inventario de especies pecuarias criadas en el agroecosistema tradicional, con el fin de generar información que coadyuve a la mejor compresión de su dinámica e importancia.

\section{METODOLOGÍA}

La información base de la presente investigación se obtuvo en cuatro localidades rurales, dos ubicadas en el estado de Yucatán y las restantes ubicadas en el estado de Campeche. Dichas localidades se seleccionaron considerando tanto su ubicación geográfica en el estado, número de familias que las integran, lejanía o cercanía de éstas a los centros urbanos, como el predominio de la lengua maya entre los habitantes. La investigación se realizó durante los meses de marzo a junio del 2017.

La primera localidad de Yucatán es Tahmuy, pertenece al municipio de Valladolid y se encuentra ubicada en la zona Oriente del estado; se localiza a $181 \mathrm{~km}$ de la ciudad de Mérida; sus coordenadas son: $20^{\circ} 45^{\prime} 33^{\prime \prime} \mathrm{N}$ y $88^{\circ} 08^{\prime} 30^{\prime \prime} \mathrm{O}$. La segunda localidad es Xoy, del municipio de Peto, en la zona Sur; se localiza a $130 \mathrm{~km}$ de la ciudad de Mérida, y sus coordenadas son: $20^{\circ} 07^{\prime} 22^{\prime \prime} \mathrm{N}$ y $88^{\circ} 58^{\prime} 15^{\prime \prime} \mathrm{O}$. La tercera y cuarta localidad son San Nicolás, municipio de Calkiní $\left(20^{\circ} 30^{\prime} 00^{\prime \prime}\right.$ de latitud norte y $90^{\circ} 15^{\prime} 00^{\prime \prime}$ de longitud oeste) y Xcupil Cacab del municipio de Hecelchakán, cuyas coordenadas geográficas son $19^{\circ} 39^{\prime} 00^{\prime \prime}$ latitud Norte y $89^{\circ} 52^{\prime} 47^{\prime \prime}$ longitud Oeste; ambas localidades se encuentran ubicadas en la zona Noreste del estado de Campeche, se localizan a 110 y $97 \mathrm{~km}$ de la capital del estado, respectivamente.Como instrumento para la obtención de información primaria, se empleó una cédula de entrevista dirigida a las jefas de familia. A partir de dicho instrumento se obtuvo información socioeconómica relevante, como número y edad de los integrantes de la familia, nivel educativo, ingresos y actividad económica; de igual manera se obtuvo información sobre el inventario, manejo y conservación de especies animales del traspatio, entre otras variables de interés.

Para la selección de las familias en Tahmuy, Xoy y Xcupil Cacab, se empleó la técnica de muestreo estadístico por conglomerados (Scheaffer y Mendahall, 1987), en dicho procedimiento se consideró a la manzana o cuadra como conglomerado y como unidades de muestreo a las familias que en ella tienen su predio. Debido al número reducido de familias por manzana, la muestra preliminar consistió en la selección al azar de un $30 \%$ del total de manzanas que integran cada una de las localidades. La variable asociada al muestreo fue la superficie del traspatio y como error de estimación el $10 \%$ del valor promedio de dicha superficie; el tamaño de la muestra final por localidad resultó inferior al preliminar.

El número de jefas de familia entrevistadas en la localidad de Tahmuy fue de 68, en Xoy este número fue de 60 y en Xcupil Cacab se entrevistaron a 55 jefas de familia. En la localidad de San Nicolás, se realizó un censo debido a su escaso número de manzanas y de familias que integran en la población; en esta localidad se entrevistaron a 27 jefas de familia. La muestra agregada en las cuatro comunidades de interés fue de 210 familias.

Los principales parámetros relacionados con las variables económicas y sociales fueron estimados utilizando estadística descriptiva, mientras que el grado de asociación entre variables de la unidad doméstica y la diversidad de especies del traspatio se estimó mediante el coeficiente de correlación de Pearson (Montgomery, 2011). La diversidad, riqueza específica, similitud y abundancia de las especies pecuarias presentes en los traspatios se estimaron mediante los índices de Shannon-Wienner, Simpson, Margalef, BergerParker, Jaccard y Equitatividad, según su importancia y área de aplicación de cada uno de ellos.

El índice de diversidad de Shannon-Wienner se estimó mediante la siguiente ecuación:

$$
H^{\prime}=\Sigma p i \ln p i
$$

Dónde: $p_{i}=$ abundancia proporcional de la $i$-ésima especie: $(n / N)$, In = logaritmo natural.

El índice de dominancia de Simpson se estimó por:

$$
D=\Sigma p i^{2}
$$

Dónde: $p_{i}=$ abundancia proporcional de la $i$-ésima especie: $\left(n_{i} / N\right)$.

El índice de diversidad de Margalef de acuerdo con Clifford y Stephenson (1975), se estimó a través de la siguiente expresión: 


$$
D_{m g}=(S-1) / \ln N
$$

Dónde: $S=$ número de especies presentes, $N=$ número total de individuos.

El índice de Berger-Parker se estimó por medio de:

$$
D=N_{\text {máx }} / N_{\text {Total }}
$$

Dónde: $N_{\text {máx }}=$ abundancia máxima, $N_{\text {Total }}=$ abundancia o densidad total.

El Coeficiente de similitud de Jaccard se estimó a través de la siguiente expresión:

$$
J=c /(a+b-c)
$$

Dónde: $\mathrm{a}=$ número de especies presentes en el sitio $\mathrm{A}$, $\mathrm{b}=$ número de especies presentes en el sitio $\mathrm{B}$ y $\mathrm{c}=$ número de especies presentes en ambos sitios $A$ y $B$.

Por último, el Índice de equidad o equitatividad de Pielou, se calculó de acuerdo a la siguiente expresión:

$$
\mathrm{J}^{\prime}=\mathrm{H}^{\prime} / \mathrm{H}_{\max }^{\prime}
$$

Dónde: $\mathrm{H}_{\max }^{\prime}=\ln (S)$ y $\mathrm{H}^{\prime}=$ medida logarítmica de la diversidad.

Toda la información obtenida se registró y proceso utilizando el programa Excel en sus diversas aplicaciones estadísticas.

\section{RESULTADOS Y DISCUSIÓN}

La información obtenida evidencia que en los agroecosistemas tradicionales las familias crían siete especies de animales, de las cuales cuatro son aves y tres pertenecen a mamíferos (Tabla 1). De las aves, se identificaron gallinas, pavos, patos y palomas; estos resultados son similares a los reportados por Guerra (2005) para la comunidad de Yaxcabá, Yucatán, donde se encontró el mismo número de especies avícolas domésticas.
La presencia de aves en el traspatio se debe a su importancia para la familia, ya que éstas son especies proveedoras de carne y huevo bajo condiciones de rusticidad, debido a que provienen de genes seleccionados en condiciones poco favorables de producción (Cuca et al., 2015). Así mismo, la alimentación animal no representa una inversión alta para las familias, y desde el punto de vista cultural, estas especies juegan un papel importante en las costumbres de las comunidades rurales, especialmente en la gastronomía de cada región.Es importante mencionar que las familias del medio rural tienen diferentes preferencias con relación a la cría de animales domésticos en el agroecosistema tradicional (Novelo et al., 2016). Por ejemplo, en el presente estudio se encontró que en la mayor parte de las familias que integran la muestra crían dos especies pecuarias, las cuales son las aves de corral y cerdos (Tabla 2); cuya producción es utilizada como fuente de alimento o de ahorro (Olguín et al., 2017). La comunidad de Xcupil Cacab fue la única en la muestra donde se encontraron cinco especies de interés (aves de corral, cerdos, ovinos, bovinos y otras aves).

Por otra parte, se encontró que las familias en las localidades estudiadas crían en promedio 22 individuos o animales, lo cual se asocia a factores como tamaño del predio, actividad económica a la que se dedican los jefes de familia y como forma de uso de los diferentes residuos de la cocina y cosecha; el número de individuos resultó superior a Tabla 2. Número promedio de especies pecuarias que crían las familias en los traspatios de la Zona Maya de Yucatán y Campeche.

Table 2. Average number of livestock species raised by families in their backyards in the Mayan Zone of Yucatan and Campeche.

\begin{tabular}{lccccc}
\hline $\begin{array}{l}\text { NÚM. DE } \\
\text { ESP. PEC. }\end{array}$ & $\begin{array}{c}\text { PROMEDIO } \\
(\%)\end{array}$ & $\begin{array}{c}\text { XOY } \\
(\%)\end{array}$ & $\begin{array}{c}\text { TAHMUY } \\
(. \%)\end{array}$ & $\begin{array}{c}\text { S. NICOLÁS } \\
(\%)\end{array}$ & $\begin{array}{c}\text { XCUPIL } \\
\text { C. }(\%)\end{array}$ \\
\hline Una esp. & 38.5 & 32.7 & 38.3 & 60.0 & 36.4 \\
\hline Dos esp. & 39.4 & 45.5 & 43.3 & 28.0 & 32.7 \\
\hline Tres esp. & 18.5 & 20.0 & 13.3 & 12.0 & 25.5 \\
\hline Cuatro esp. & 3.1 & 1.8 & 5.1 & 0.0 & 3.6 \\
\hline Cinco esp. & 0.5 & 0.0 & 0.0 & 0.0 & 1.8 \\
\hline
\end{tabular}

Fuente: Encuesta realizada a familias en 2017.

Tabla 1. Familias de la Zona Maya de Yucatán y Campeche que crían especies pecuarias en el traspatio.

Table 1. Mayan Zone families in Yucatan and Campeche that breed livestock species in the backyard.

\begin{tabular}{lccccc}
\hline $\begin{array}{l}\text { ESPECIE } \\
\text { PECUARIA }\end{array}$ & PROMEDIO(\%) & XOY(\%) & TAHMUY(.\%) & $\begin{array}{c}\text { S. NICOLÁS } \\
(\%)\end{array}$ & $\begin{array}{c}\text { XCUPIL C. } \\
(\%)\end{array}$ \\
\hline Gallinas $^{1}$ & 82.6 & 74.5 & 86.7 & 88.0 & 83.6 \\
\hline Pavos $^{2}$ & 36.4 & 20.0 & 36.7 & 32.0 & 54.5 \\
\hline Otras aves $^{3}$ & 5.6 & 7.3 & 6.7 & 0.0 & 5.5 \\
\hline Ovinos & 7.2 & 9.1 & 1.7 & 8.0 & 10.9 \\
\hline Cerdos & 53.8 & 76.4 & 53.3 & 20.0 & 47.3 \\
\hline Bovinos & 1.0 & 3.6 & 0.0 & 0.0 & 0.0 \\
\hline
\end{tabular}

Nota: 1. Incluye pollitos, pollos en crecimiento y gallos.

2. Incluye pavitos, pavos en crecimiento y pavas pie de cría.

3. Incluye patos y palomas.

Fuente: Encuesta realizada a familias en 2017. 
lo reportado por Rejón et al., (1996) en Texán y Tzacalá y por Guerra (2005) en Yaxcabá, localidades del estado de Yucatán, donde se crían 16, 12 y 18 animales, respectivamente.

La cría de bovinos se realiza solo en algunos traspatios y debido al tamaño de los individuos solo son manejados por el jefe de familia, pero como éste mayormente migra a centros urbanos en busca de oportunidades de trabajo, el porcentaje de familias que tiene en su inventario animal a esta especie resultó muy bajo (Tabla 3). Situación similar fue reportada por Rodríguez et al. (2011), cuyo estudio lo realizaron tanto en localidades indígenas de la región Altos de Chiapas, como en colonias con población indígena localizadas en el sector peri-urbano de la ciudad de San Cristóbal de Las Casas, donde los bovinos son la especie menos representativa en los traspatios. Así mismo, en una investigación realizada por Gutiérrez et al. (2012) en 33 comunidades del estado de Yucatán, determinaron que solo el $0.9 \%$ de las familias criaban la citada especie.

Tabla 3. Inventario promedio de especies pecuarias criadas por las familias en los traspatios de la Zona Maya de Yucatán y Campeche.

Table 3. Average inventory of livestock species raised by families in their backyards in the Mayan Zone of Yucatan and Campeche.

\begin{tabular}{lccccc}
\hline $\begin{array}{l}\text { ESPECIE } \\
\text { PECUARIA }\end{array}$ & PROMEDIO & XOY & TAHMUY & S. NICOLÁs & $\begin{array}{c}\text { XCUPIL } \\
\text { C. }\end{array}$ \\
\hline $\begin{array}{l}\text { Aves de } \\
\text { corral (\%) }\end{array}$ & 84.1 & 78.2 & 86.7 & 92.0 & 83.6 \\
\hline Inventario & 20.1 & 21.2 & 17.2 & 15.5 & 24.6 \\
\hline Ovinos (\%) & 7.2 & 9.1 & 1.7 & 8.0 & 10.9 \\
\hline Inventario & 6.1 & 3.0 & 5.0 & 4.0 & 9.5 \\
\hline Cerdos (\%) & 53.8 & 76.4 & 53.3 & 20.0 & 47.3 \\
\hline Inventario & 2.5 & 2.6 & 2.1 & 4.0 & 2.5 \\
\hline Bovinos (\%) & 1.0 & 3.6 & 0.0 & 0.0 & 0.0 \\
\hline $\begin{array}{l}\text { Inventario } \\
\text { Otras aves }\end{array}$ & 1.0 & 1.0 & 0.0 & 0.0 & 0.0 \\
(\%) & 5.6 & 7.3 & 6.7 & 0.0 & 5.5 \\
\hline Inventario & 17.0 & 8.8 & 30.8 & 0.0 & 9.7 \\
\hline
\end{tabular}

Fuente: Encuesta realizada a familias en 2017.

Con base en la información de la Tabla 3, se puede afirmar que las familias rurales de Yucatán y Campeche donde fue realizada la investigación, presentan diferencias en la estructura de su inventario animal, en la que destacan las aves de corral, ya que el $84.1 \%$ de las familias optan por su cría. Trabajos realizados por Gutiérrez et al. (2007), Guerra (2005) y Rejón et al. (1996) en los municipios de Tetiz y Yaxcabá, Yucatán, y en comisarías de Texán y Tzacala de la ciudad de Mérida del mismo estado, reportaron porcentajes de cría similares ( $82.8,85,81$ y $84 \%$, respectivamente). Sin embargo, estos resultados difieren con lo publicado por Vargas et al. (2012) para varias regiones del estado de Puebla, donde reportan que la especie animal más representativa son los caprinos (46.6\%); mientras que en Yucatán y Campeche dicha especie no fue reportada como parte del inventario animal del traspatio.
Cabe destacar que la presencia de los cerdos en los agroecosistemas tradicionales se ubica en segundo lugar de importancia, los cuales son criados por el $53.8 \%$ de las familias de la muestra. En la comunidad de Xoy se registró la mayor presencia de tales animales (76.4\%), debido a que esta especie significa para las familias una fuente de ingresos y ahorro, lo cual se materializa cuando los jefes de familia venden dichos animales en situaciones de necesidad económica, como pago de deudas, compra de medicamentos por enfermedad de algún familiar, adquisición de bienes de consumo, o simplemente engordan los cerdos para contribuir a las festividades familiares o patronales de la comunidad. Estudios realizados por Vargas et al. (2017) en la región de la Montaña del estado de Guerrero, encontraron que la presencia de los cerdos se ubica en la cuarta posición del inventario animal de las familias (4.8\%), por lo que sus resultados difieren con lo encontrado en este trabajo.

Por su parte, en la comunidad de San Nicolás se encontró que el inventario porcino de las familias que crían cerdos fue de cuatro individuos, resultado del programa académico "Hombre en la Tierra" dirigido por la Universidad Anáhuac del Mayab, ubicada en la ciudad de Mérida, mismo que benefició a las familias durante el periodo de 2015 a 2016. Este programa se orientó hacia la reproducción y engorda de cerdos pelón mexicano, con el fin de contribuir a la conservación de este recurso zoogenético.Con relación a la cría de aves de corral que se practica en el traspatio de las cuatro comunidades de interés (Tabla 4), se considera a ésta como una actividad relevante y es realizada por la mayoría de las familias (84.1\%); esto principalmente por la baja inversión que se requiere para la alimentación de dichos animales (desperdicios de cocina, masa o tortilla), y porque se dispone en parte del maíz cosechado en la milpa para este fin. Otro factor importante relacionado con la citada cría, en comparación con otras especies pecuarias, es el corto tiempo o periodo de crecimiento y reproducción de las aves, así como por la facilidad de manejo y explotación de las mismas

Tabla 4. Inventario promedio de aves de corral en el traspatio familiar de la Zona Maya de Yucatán y Campeche.

Table 4. Average inventory of poultry at family backyard in the Mayan Zone of Yucatan and Campeche.

\begin{tabular}{lccccc}
\hline ANIMAL & PROMEDIO & XOY & TAHMUY & S.NICOLÁS & XCUPIL \\
\hline $\begin{array}{l}\text { Pollo } \\
\text { engorda }\end{array}$ & 12.3 & 6.0 & 5.7 & 6.0 & 10.0 \\
\hline Pollitos & 10.6 & 10.8 & 9.1 & 8.4 & 13.1 \\
\hline Gallina & 10.7 & 9.8 & 10.4 & 9.4 & 12.6 \\
\hline Gallo & 1.8 & 2.0 & 1.6 & 1.2 & 2.1 \\
\hline $\begin{array}{l}\text { Pavitos } \\
\text { Pava }\end{array}$ & 6.4 & 6.8 & 2.0 & 2.0 & 7.6 \\
\hline $\begin{array}{l}\text { Pavo } \\
\text { engorda }\end{array}$ & 3.2 & 2.8 & 3.8 & 1.5 & 3.3 \\
$\begin{array}{l}\text { Pavo pie } \\
\text { de cría }\end{array}$ & 2.1 & 3.5 & 3.0 & 7.4 & 2.3 \\
\hline
\end{tabular}

Fuente: Encuesta realizada a familias en 2017. 
(Gutiérrez et al., 2007).Las familias del medio rural disponen de un inventario promedio de aves de corral conformado mayormente por pollos de engorda y gallinas, cuyo número promedio fue de 12.3 y 10.7 individuos, respectivamente. En un estudio realizado por Macdonal et al. (2015) en "La Ventana", Chamula, Chiapas, reportaron que la parvada cuenta en promedio con seis gallinas, por lo que dichos resultados contrastan con lo obtenido en el presente trabajo. Por su parte, en la comunidad de Xcupil Cacab fue donde se registró el mayor inventario de aves de corral, hecho determinado por su lejanía a algún centro urbano o de abastecimiento de alimentos, tal y como lo reportaron Gutiérrez et al. (2007) en su estudio realizado en el municipio de Tetiz, Yucatán, donde concluyeron que las diferencias en el promedio de animales criados por familia puede estar afectadas por la cercanía a la ciudad de Mérida.

Las aves que tienen menos presencia en el inventario familiar son los gallos (1.8 individuos), ya que éstos solo son utilizados en la mayor parte de los traspatios como píes de cría y, una vez que éstos dejan de ser fértiles o poco productivos, son reemplazados por nuevos animales; debido a que la reproducción es su única función y su carne es poco preferida.

Los cerdos, después de las aves de corral, son el recurso zoogenético con mayor presencia de individuos en los traspatios de los hogares encuestados, su importancia se debe principalmente por los ingresos que genera, ya sea por su venta en pie o en carne (López et al., 2012); en la Tabla 5 se observa la diversidad de su genotipo. Estos animales se encuentran presentes en el $53.8 \%$ de los agroecosistemas tradicionales, en el que predomina la presencia de individuos provenientes de la cruza de diferentes razas $y$, en menor proporción, se observó el genotipo comercial o mejorado. En una investigación realizada por Gutiérrez et al. (2012) en 33 comunidades rurales del estado de Yucatán, encontraron que las familias que criaban cerdos tenían mayor preferencia por los animales provenientes de cruzas $(79.5 \%)$, mientras que una menor proporción criaban animales del tipo comercial ( $16.9 \%$ y solo una mínima parte de éstas criaban ambos tipos de cerdos (3.6\%).

Tabla 5. Familias que crían cerdos en el traspatio y su inventario promedio por genotipo en la Zona Maya de Yucatán y Campeche.

Table 5. Families that raise pigs in their backyard and their average inventory by genotype in the Mayan Zone of Yucatan and Campeche.

\begin{tabular}{lccccc}
\hline $\begin{array}{l}\text { TIPO DE } \\
\text { CERDO }\end{array}$ & PROMEDIO & XOY & TAHMUY & S. NICOLÁS & XCUPIL \\
\hline $\begin{array}{l}\text { Mejorado } \\
\text { (\%) }\end{array}$ & 5.6 & 7.3 & 6.7 & 12.0 & 0.0 \\
\hline Inventario & 1.9 & 1.3 & 2.0 & 2.7 & 0.0 \\
\hline Cruza (\%) & 35.4 & 43.6 & 45.0 & 0.0 & 32.7 \\
\hline Inventario & 2.4 & 3.1 & 1.9 & 0.0 & 2.3 \\
\hline Pelón (\%) & 19.0 & 36.4 & 8.3 & 8.0 & 18.2 \\
\hline Inventario & 2.1 & 1.6 & 1.6 & 6.0 & 2.4 \\
\hline
\end{tabular}

Fuente: Encuesta realizada a familias en 2017.
La alimentación de los cerdos en el traspatio consistió mayormente de masa diluida en agua (atole), maíz en grano y desperdicios de cocina, productos que no representan un gasto significativo para las familias; resultados que coinciden con lo encontrado por Mendoza et al. (2014) en localidades del municipio de San Lucas, Chiapas. Sin embargo, para el genotipo comercial o mejorado se observó que las familias suministran alimento balanceado y, por el valor de este insumo, las familias optan posteriormente por criar un genotipo mejor adaptado al medio; otro factor que determina que un limitado número de familias críen este genotipo comercial son las enfermedades, ya que los cerdos mejorados son más propensos a padecer diversos males, caso contrario ocurre con los animales que provienen de diferentes cruzas, los que presentan una mayor variabilidad genética $y$, en consecuencia, tolerancia a las variables ambientales extremas y resistencia a ciertos parásitos, lo que garantiza una elevada tasa de supervivencia para los animales provenientes de cruzas (Scarpa et al., 2003).En lo que respecta a la diversidad, abundancia, similitud y riqueza de especies animales que integran los agroecosistemas tradicionales en las comunidades mayas de Yucatán y Campeche estudiadas (Tabla 6), dependen en gran medida de múltiples condiciones como la forma de aprovechamiento de las mismas, nivel de ingreso, tradición cultural, preferencias en consumo, lejanía de las comunidades a los centros urbanos o de abasto, excedentes de cosecha de la milpa o traspatio y disponibilidad de residuos de alimentos del hogar. Como ya se ha mencionado, la cría de diversas especies animales es una característica de las familias del medio rural, ya que de esta manera se garantiza la producción y disponibilidad de alimentos, la existencia de una fuente marginal de ingreso, ahorro y conservación de los recursos zoogenéticos (Salazar et al., 2015).El primer

Tabla 6. Índices de diversidad de las especies pecuarias criadas por las familias en el traspatio de la Zona Maya de Yucatán y Campeche.

Table 6. Indices of livestock species diversity reared by families in the backyard of the Mayan Zone of Yucatan and Campeche.

\begin{tabular}{lccccc}
\hline ÍNDICE & PROMEDIO & XOY & TAHMUY & S. NICOLÁS & $\begin{array}{c}\text { XCUPIL } \\
\text { C }\end{array}$ \\
\hline Shannon & 0.903 & 0.806 & 0.895 & 0.694 & 0.947 \\
Simpson & 0.565 & 0.619 & 0.555 & 0.656 & 0.520 \\
\hline Margalef & 0.606 & 0.717 & 0.715 & 0.840 & 0.698 \\
Berger Parker & 0.736 & 0.776 & 0.727 & 0.798 & 0.692 \\
\hline Jaccard & $0.833^{1}$ & $1.000^{2}$ & $0.800^{3}$ & $0.600^{4}$ & $0.800^{5}$ \\
\hline Equitatividad & 0.504 & 0.581 & 0.646 & 0.632 & 0.588 \\
\hline
\end{tabular}

Nota:

1. Compara las dos comunidades de Yucatán, con las dos comunidades de Campeche.

2. Compara las comunidades de Xoy y Tahmuy, Yucatán.

3. Compara las comunidades de Tahmuy, Yucatán y Xcupil Cacab, Campeche.

4. Compara las comunidades de Sn. Nicolás y Xcupil Cacab, Campeche.

5. Se comparó la Comunidad de Xcupil Cacab, Campeche con la comunidad de Xoy, Yucatán.

Fuente: Encuesta realizada a familias en 2017 
índice de diversidad, el de Shannon-Wiener, mide la diversidad específica y cuyo valor estimado al ubicarse por debajo de la unidad evidencia que en las comunidades de interés el inventario animal presenta una baja diversidad, esto debido a que la mayoría de las familias optan generalmente por criar aves de corral y cerdos, especies de las que obtienen mayores beneficios en corto plazo y durante la mayor parte del año, como la disponibilidad de huevos de ave.

El segundo índice de diversidad, el de Simpson, demuestra que en la comunidad de San Nicolás existe la dominancia de una especie (aves de corral), situación que se asocia a las ventajas que ofrece ésta por su función multipropósito como la producción de carne, huevo y pie de cría. De igual manera, las aves de corral cumplen funciones culturales y ecológicas, esto último por el aporte de nutrientes mediante sus excretas que se emplean como abono para los cultivos del agroecosistema tradicional (Camacho et al., 2006; Solórzano et al., 2014;). Es importante mencionar que en cada una de las comunidades de la muestra se presenta la dominancia de una sola especie, observación que es confirmada por el índice de Berger Parker, ya que con éste se constató la dominancia de una especie y una menor diversidad. Esta dominancia la presentan las aves de corral, debido a sus características ya descritas.Con el fin de comparar el grado de semejanza por presencia de especies animales entre comunidades, se estimó el índice de Jaccard, (Magurran 1988), y a través de éste se constató que en las comunidades del estado de Yucatán existe semejanza en la diversidad de especies que crían las familias en sus traspatios. Esta práctica está determinada por las costumbres en el estado y esto se demuestra por las preferencias que tienen las familias hacia determinadas especies pecuarias. Caso contrario se encontró entre las comunidades de Campeche, donde el grado de semejanza fue menor.

Con base en el valor estimado del índice de Equitatividad, que mide el grado de similitud de las especies por su respectiva abundancia (López y Marone, 1996), se comprobó que la comunidad de Tahmuy es la que presenta el mayor grado de equitatividad, ya que se constató que la mayoría de las familias poseen un número similar de individuos por cada especie presente en el inventario animal; característica determinada por factores como el tamaño del predio, excedentes de la producción agrícola, arraigo a las fiestas patronales y el beneficio que obtienen las familias de las especies animales que crían en el traspatio.

Por su parte, es importante señalar que las principales variables socioeconómicas que caracterizan a las familias mayas de Yucatán y Campeche, son relevantes en la compresión de la diversidad pecuaria presente en los traspatios de las localidades estudiadas. En general, se encontró que la unidad familiar está conformada en promedio por 3.7 integrantes (padre, madre, hijos, parientes), el padre es quien posee la mayor edad promedio (54.6 años), la madre cuenta con 50.5 años, mientras que los hijos tienen 17.4 años y los parientes cercanos que habitan en el mismo predio que la familia nuclear poseen 27.1 años de edad.
La mayor parte de los integrantes de la familia no concluyó el sexto año de primaria. El padre tiene en promedio una escolaridad de 4.4 años, la madre 4.2 años, los hijos 7.7 años y los parientes 5.6 años. Lo anterior indica que las nuevas generaciones (los hijos) presentan una escolaridad mayor a la de los padres, pero no alcanzan concluir la educación secundaria, la cual es de nueve años de instrucción formal. Así mismo, se encontró que la mujer es quién se encargada de realizar las principales actividades del traspatio familiar; la mayor parte de su tiempo lo destina a la alimentación y crianza de los animales domésticos. Con respecto a la superficie del traspatio, éste posee una extensión promedio de $617.9 \mathrm{~m}^{2}$ en las comunidades de interés, lo cual favorece las actividades productivas que se desarrollan para el consumo familiar, venta de excedentes, intercambio y regalo.

Con relación al fenómeno de la migración rural, se encontró que el $16.1 \%$ de los padres, el $18.2 \%$ de los hijos y solamente el $3.1 \%$ de las madres migran de la comunidad hacia diferentes centros urbanos. El tipo de migración es el pendular y el temporal, cuyos principales destinos son las capitales de los estados o las zonas turísticas; la principal razón de esta migración es con el fin de buscar un empleo remunerado que no se ofrece en sus comunidades de origen. Sin embargo, se observó que una parte de los padres de familia que migran retornan a la comunidad cuando es época de siembra y cosecha de maíz en sus milpas.

En las comunidades de estudio, el ingreso anual de la unidad familiar es en promedio de 49,452.7 pesos mexicanos. Cuya estructura la componen en un $88.4 \%$ los salarios de los integrantes de la familia, los cuales en su mayoría laboran fuera de la comunidad; los apoyos que otorga el gobierno como PROSPERA, PROCAMPO, pensión para adultos mayores, entre otros, aportan el $6.2 \%$ del ingreso. Por su parte, el valor de la venta de los productos pecuarios contribuye con el $4.4 \%$ al ingreso familiar; mientras que las remesas (0.7 \%) y la venta de productos agrícolas del traspatio $(0.3 \%)$, son las que contribuyen en menor proporción al citado ingreso.

Respecto a la interacción que se establece entre las variables socioeconómicas que caracterizan a las familias de la muestra con la diversidad de especies pecuarias en el traspatio, se determinó que la edad de la madre presenta una relación directa con la citada diversidad (Tabla 7), situación que evidencia el arraigo a las tradiciones culturales en las comunidades mayas. Por ejemplo, se encontró que mientras más avanzada sea la edad de la madre, la diversidad de especies pecuarias es mayor; caso contrario ocurre con la escolaridad y la migración. Estos dos últimos factores se asocian en forma inversa con la diversidad de las especies, debido a que la menor edad de las jefas de familia permite mayores oportunidades de formación educativa y laborales fuera de la comunidad; por lo que éstas y algunos miembros de la familia no cuentan con el tiempo suficiente para la cría de especies pecuarias; fenómeno que favorece la disminución de la diversidad y el abandono gradual de la actividad pecuaria productiva en el traspatio. 
Tabla 7. Coeficientes de correlación entre variables socioeconómicas y la diversidad de especies pecuarias en la Zona Maya de Yucatán y Campeche.

Table 7. Correlation coefficients between socioeconomic variables and the diversity

of livestock species in Mayan communities of Yucatan and Campeche.

\begin{tabular}{|c|c|c|c|c|c|c|c|}
\hline & $\begin{array}{l}\text { SUPERFICIE } \\
\text { PREDIO }\end{array}$ & $\begin{array}{l}\text { EDAD } \\
\text { MADRE }\end{array}$ & $\begin{array}{l}\text { ESCOL. } \\
\text { MADRE }\end{array}$ & $\begin{array}{l}\text { INTEGRANT. } \\
\text { FAMILIA }\end{array}$ & $\begin{array}{l}\text { INGRESO } \\
\text { ANUAL }\end{array}$ & MIGRACIÓN & $\begin{array}{c}\text { ÍNDICE } \\
\text { SHANNON }\end{array}$ \\
\hline SUP. PREDIO & 1.0000 & & & & & & \\
\hline EDAD MADRE & 0.1090 & 1.0000 & & & & & \\
\hline ESCOL. MADRE & -0.1196 & -0.6058 & 1.0000 & & & & \\
\hline INT. FAMILIA & 0.0460 & -0.3548 & 0.2748 & 1.0000 & & & \\
\hline INGRESO FAM & 0.0366 & -0.2183 & 0.3501 & 0.4936 & 1.0000 & & \\
\hline MIGRACIÓN & 0.0366 & -0.2814 & 0.2349 & 0.4239 & 0.6982 & 1.0000 & \\
\hline IND. SHANNON & 0.3269 & 0.1685 & -0.0839 & -0.0878 & 0.0176 & -0.1110 & 1.0000 \\
\hline
\end{tabular}

Fuente: Encuesta realizada a familias en 2017.

Por último, se encontró que existe una relación directa entre el tamaño del predio y la diversidad de especies pecuarias presentes en el agroecosistema tradicional, relación que se debe principalmente a la disponibilidad de mayores espacios para el manejo apropiado de las mismas y por la interacción favorable entre las especies pecuarias y los cultivos agrícolas de bajo porte, como hortalizas, ornamentales y medicinables. Es importante mencionar que debido a la limitada disponibilidad de información proveniente de investigaciones relativas a la interacción entre las variables socioeconómicas y la diversidad de especies pecuarias que se crían en el traspatio, la discusión de los resultados obtenidos en el presente no fue posible contrastarlos por lo ya indicado.

\section{CONCLUSIONES}

El agroecosistema tradicional o traspatio en las comunidades mayas de Tahmuy y Xoy, en el estado de Yucatán, así como en Xcupil Cacab y San Nicolás, estado de Campeche, presenta una baja diversidad de especies pecuarias, debido a que predominan las aves de corral y los cerdos de genotipo no mejorado. Entre los principales factores socioeconómicos que influyen sobre la diversidad y estructura del inventario animal en el traspatio del área estudiada, destacan en forma directa el tamaño del predio y la edad de la jefa de familia, no así el nivel de escolaridad de ésta y la migración.

La cría de animales en el traspatio ofrece en forma temporal productos que son destinados a la alimentación de la familia, para festejos, intercambio y venta. Por último, es importante mencionar que las mujeres de estas comunidades rurales son quienes generalmente se encargan del manejo y aprovechamiento de las especies pecuarias, esto como resultado del conocimiento adquirido, tradición y costumbres de la región, así como de la disponibilidad de su tiempo.

\section{AGRADECIMIENTOS}

Al CONACYT, por el financiamiento otorgado al proyecto de investigación "Conservación del Cerdo Pelón Mexi- cano: Estrategias de Producción Sustentable para la Zona Maya de la Península de Yucatán", Clave: 248961; de cuyos resultados forma parte el presente artículo.

\section{REFERENCIAS}

Aké, G.A., Ávila, M., Jiménez, O.J. 2002. Valor de los productos directos del agroecosistema solar: El caso de Hocabá, Yucatán, México. Sociedades rurales, producción y medio ambiente. México. 3 (1). 7-18.

Camacho, E. M. A., Lira, T. I., Ramírez, C. L., López, P. R., Arcos, G. J. L. 2006. La avicultura de traspatio en la costa de Oaxaca, México. Ciencia y Mar. Universidad del Mar. Puerto Ángel, Pochutla, Oaxaca. 10 (28). 3-11.

Cuanulo de la C.H.E., Guerra, M.R.R. 2009. La estructura del grupo doméstico y del solar como ecosistemas en una comunidad mayense de Yucatán. X Congreso Internacional. V Congreso anual de Agricultura Sostenible.Cuca, G. J. M., Gutiérrez, A. D. A., López, P. E. 2015. La avicultura de traspatio en México: Historia y caracterización. Agroproductividad. Colegio de Postgraduados, Campus Montecillo, Montecillo, Estado de México. México. 8 (4). 30-36.

Gliessman, S. 1990. Understanding the basis for sustainability of agriculture in the tropics: experiences in Latin America. In: Edwards, et. al. (ed), Sustainable Agricultural Systems. Soil \& Water Conservation Society. Ankeny, lowa.

González, O.F., Pérez, M.A., Ocampo, F.I., Paredes, S.J.A., De la Rosa, P.P. 2014. Contribuciones de la producción en traspatio a los grupos domésticos campesinos. Estudios Sociales. Coordinación de Desarrollo Regional. Hermosillo, México 22 (44). 145-170.

Guarneros, Z.N., Morales, J.J., Cruz, H.J., Huerta, P.A., Ávalos, C.D.A. 2014. Economía familiar e índice de biodiversidad de especies en los traspatios comunitario de Santa María Nepopualco, Puebla. Revista Mexicana de Ciencias Agrícolas. Instituto Nacional de Investigaciones Forestales, Agrícolas y Pecuarias. Estado de México, México. (9). 1701-1712.

Guerra M.R. 2005. Factores sociales y económicos que definen el sistema de producción de traspatio en una comunidad rural de Yucatán, México. Tesis de Maestría en Ciencias. Centro de Investigaciones y Estudios Avanzados del Instituto Politécnico. Nacional. Unidad Mérida, Departamento de Ecología Humana. 97 p. 
Gutiérrez, R.E.J., Aranda, C.F.J., Rodríguez, V.R.I., Bolio, G.M.E., Ramírez, G.S., Estrella,T.J. 2012. Factores sociales de la crianza de animales de traspatio en Yucatán. Bioagrociencias. 5 (1). 20-28.

Gutiérrez, T.M.A., Segura, C.J.C., López, B.L., Santos, F.J., Santos R.R.H., Sarmiento, F.L., Carvajal, H.M., Molina, C.G. 2007. Características de la avicultura de traspatio en el Municipio de Tetiz, Yucatán, México. Tropical and Subtropical Agroecosystems. Vol. 7. Núm. 3. Universidad Autónoma de Yucatán. Mérida, Yucatán, México. pp. 217-224.

López de C.J., Marone, L. 1996. Efectos de la riqueza y de la equitatividad sobre los valores de diversidad en comunidades de aves. Ecología. Buenos Aires, Argentina. (10). 447-455.

López, G.J.L., Damián, H.M.A.; Álvarez, G.F., Parra, I.F., Zuluaga, S.G.P. 2012. La economía de traspatio como estrategia de supervivencia en San Nicolás de los Ranchos, Puebla, México. Revista de Geografía Agrícola. Universidad Autónoma Chapingo. Texcoco, México. (48-49). 51-62.

Macdonal, J.M., Zaragoza, L., Rodríguez, G. 2014. Función de los animales del traspatio en "la Ventana" (Chamula Chiapas) como apoyo a la familia. AICA. Actas Iberoamericanas de Conservación Animal. pp. 184-189.

Magurran, A.E. 1988. Ecological diversity and its measurement. Princenton University Press.

Mariaca, M.R. 2012. La complejidad del huerto familiar maya del Sureste de México, en: Mariaca, M., R. (Eds.) El huerto familiar del Sureste de México. Secretaria de Recursos Naturales y Protección ambiental del estado de Tabasco, Colegio de la Frontera Sur. México. 544 p.

Mendoza, L., Zaragoza, L., Rodríguez, G. 2014. Caracterización del componente pecuario del traspatio en localidades del municipio de San Lucas, Chiapas, México. AICA. Actas Iberoamericanas de Conservación Animal. 4. 219-221.

Montgomery, D.C. 2011. Diseño y análisis de experimentos. Segunda edición. Editorial Limusa Wiley. México. p 81.

Novelo, P.D., Magaña, M.M., Sierra, V.A. 2016. Manejo y aprovechamiento de especies pecuarias criadas en el traspatio en comunidades rurales de Yucatán, México. AICA. Actas Iberoamericanas de Conservación Animal. 24-32.

Olguín, P.C., Álvarez, A.M. del C., Asiain, H.A. 2017. Investigación aplicada para manejo de recursos naturales del solar familiar tropical. Agroproductividad. Colegio de Postgraduados. Montecillo, Texcoco, Estado de México. 10 (7). 9-14.Olvera, H.J.I., Álvarez, C.N.M., Aceves, R.E., Guerrero, R.J. de D. 2017. Perspectivas del traspatio y su importancia en la seguridad alimentaria. Agroproductividad. Colegio de Postgraduados. Montecillo, Texcoco, Estado de México. 10 (7). 39-45.Rejón, A.M.J., Dájer, A.A.F., Honhold, N. 1996. Diagnóstico comparativo de la ganadería de traspatio en las comunidades Texán y Tzacalá de la zona henequenera del Estado de Yucatán. Veterinaria México. 27. 49-55.

Rodríguez, G.G., Perezgrovas, G.R., Zaragoza, M.L. 2011. El traspatio como espacio de empoderamiento para la mujer tzotzil en Chiapas (México). AICA. Actas Iberoamericanas de Conservación Animal. 280-283.

Salazar, B.L.L., Magaña, M.M.A., Latorniere, M.L. 2015.Importancia económica y social de la agrobiodiversidad de traspatio en una comunidad rural de Yucatán, México. Agricultura, Sociedad y Desarrollo. Colegio de Postgraduados, Texcoco. México. 12 (1). 1-14.

Scarpa, R., Drucker, A., Anderson, S., Ferraes, E.N., Gómez, V., Risopatrón, R.C., Rubio, L.O. 2003. Valuing genetic resources in peasant economies: the case of 'hairless' creole pigs in Yucatan. 45. 427-443.

Scheaffer, R.L., Mendenhall y O.L. 1987. Elementos de Muestreo. Grupo Editorial Iberoamérica. Ciudad de México. México. pp 77-88.

Solórzano, M., Florio-Luis de P.J., Tamasaukas, R., Sánchez, A., Rodríguez, M., Ostos, M., Pérez, L. 2014. Manejo de aves en sistemas de avicultura familiar en la República Bolivariana de Venezuela. AICA. Actas Iberoamericanas de Conservación Animal. 4. 257-259.

Vargas, L.S., Bustamante, G.A.; Vargas, M.J., Hernández, Z.J.S., Vázquez, M.I., Calderón, S.F. 2017. Diversidad y prácticas de crianza de animales domésticos en traspatios de comunidades indígenas en Guerrero, México. Agroproductividad. Colegio de Postgraduados. Montecillo, Texcoco, Estado de México. 10 (7). 15-20. 